\title{
Individual differences in respiratory- heart period coupling and heart period responses during two attention-demanding tasks
}

\author{
S. W. PORGES and M. G. H. COLES \\ University of Illinois at Urbana-Champaign, Champaign, Illinois
}

\begin{abstract}
Respiratory-heart period coupling was assessed via the weighted coherence $\left(\mathrm{CW}_{\mathrm{W}}\right)$ statistic derived from cross-spectral analysis and was related to physiological response patterns during two attention-demanding tasks. Subjects with a high coherence responded with significantly larger heart period decelerations at the time of the imperative stimulus in a reaction-time task. Peak deceleration of the low-coherence subjects exhibited more temporal variability. In a vigilance task, individual differences in coherence assessed prior to task manipulations were maintained during the task and significantly decreased during the task. Individual differences in coherence between and within sessions were stable, whereas corresponding analyses for heart period, heart period variance, and spontaneous electrodermal activity reflected only significant within-session correlations. The data suggest that individual differences in respiratory-heart period coherence are stable and are related to phasic cardiac responses in a reaction-time task.
\end{abstract}

It has been proposed that respiratory-heart period coupling reflects an individual difference in nervous system organization related to cognitive processes such as sustained attention (Porges, 1980; Porges, Bohrer, Keren, Cheung, Franks, \& Drasgow, 1981). In the development of this hypothesis, the coupling between respiration frequency and heart period patterns was evaluated in children with attentional deficits who were treated with methylphenidate. Coupling increased only after administration of the dose of methylphenidate most consistently found to facilitate cognitive performance.

The degree of coupling between respiration and heart period is based upon a physiological relationship between phase of respiration and vagal efferent influence on the heart. Inspiration blocks vagal efferent influences to the heart and is associated with heart rate acceleration. Expiration enhances the vagal influence on the heart and is associated with heart rate deceleration. Although the specific physiological pathways mediating the "gating" of vagal activity by respiration are not known, it is known that the stretch receptor afferents in the lungs are prepotent in the triggering of the gate (see Porges, McCabe, \& Yongue, 1982). In fact, when lung inflation stimulates the stretch afferents, virtually all vagally mediated reflexes on the heart (e.g.,

This research was supported in part by NIMH Research Scientist Development Award K02-MH-0054 and National Institute of Education Grant NE-G-00-3-0013 awarded to Stephen W. Porges. Requests for reprints should be sent to Stephen W. Porges, Department of Psychology, 603 E. Daniel, University of Illinois, Champaign, Illinois 61820. chemoreceptor and baroreceptor) are blocked (see Lopes \& Palmer, 1976).

Cross-spectral analysis provides a statistic, coherence, that is a quantitative estimate of the coupling between any two sequential series on any given frequency (Brillinger, 1975). Since respiratory rhythms occur over a band of frequencies, the calculation of a summary statistic that describes the proportion of shared variance of the two systems is complex. However, a weighted coherence function $\left(\mathrm{C}_{\mathrm{W}}\right)$ can be calculated to provide an exact measurement of the proportion of variance shared by respiration and heart period within the frequency band characteristic of respiration (see Porges, Bohrer, Cheung, Drasgow, McCabe, \& Keren, 1980).

Little is known about the physiological mediation of the synchrony between respiration and heart period patterns, although the anatomical locus of the mediation must involve brainstem structures. Functionally, the coupling could be "tight," as characterized by a constant phase lag, or it could be "loose," as characterized by phase-lag "jitter" between the two physiological processes. Porges et al. (1981) described a hypothetical mechanism to explain individual differences in coupling. They proposed that the coupling could be modeled on a random time-delay generator which modulated the co-occurrence of the two systems. If the information from the stretch afferents were transformed in the brainstem by adding a random time delay before it "gated" the vagal efferent activity to the heart, then the coupling could be "loose" even though the frequency characteristics of heart period and respiration might be similar. Potentially, if a time-delay 
generator were a general characteristic of an individual's brainstem, it could be causal in the coordination of many behaviors and physiological systems that depend upon the transmission of information through the brainstem. It is possible that greater unpredictability in the time delay (e.g., a white-noise distribution) between sensory and motor communication within the nervous system may be characteristic of an individual who has deficits in attention. Moreover, since conditioning is facilitated by temporal contiguity, a greater unpredictability in this time delay may characterize individuals who are resistant to conditioning manipulations.

The present study was conducted to evaluate, in a normal population of college students not exhibiting pathologies of attention, the relationship between individual differences in weighted coherence $\left(\mathrm{CW}_{\mathrm{W}}\right)$ and physiological activity during two attentiondemanding tasks. The first, a go/no-go reaction-time task, required the subjects to respond to an imperative stimulus only on trials for which the warning stimulus had specific characteristics. The second, a vigilance task, required the subject to maintain a constant level of alertness in order to detect infrequently occurring target events. The stability of $\mathrm{CW}_{\mathrm{W}}$ between and within experimental sessions and the relationships among $\mathrm{CW}_{\mathrm{W}}$, spontaneous electrodermal activity, heart period, and heart period variability were also evaluated.

\section{METHOD}

\section{Subjects}

Twenty male undergraduate students at the University of Illinois served as the subjects. They were paid for each of the two sessions in which they participated.

\section{Apparatus and Procedure}

Prior to each experimental session, the subjects were prepared for recording of cardiac, respiratory, and electrodermal activity. All measures were recorded using a Beckman R-411 dynagraph, and cardiac activity and respiratory activity were also recorded on a Hewlett-Packard 3960 FM tape recorder for subsequent quantification by an IBM 1800 computer.

Cardiac activity (EKG) was obtained with standard-size electrodes (Beckman) located in the Lead II position and a Beckman 9852A coupler. For respiratory activity, a Beckman strain gauge transducer belt was placed above the lower rib cage and connected to a Beckman 9852A coupler in the bridge mode. For electrodermal activity, standard-size Beckman Biopotential Ag$\mathrm{AgCl}$ electrodes were placed on the volar surfaces of the index and middle fingers of the nondominant hand. A 9842 coupler, which imposes a constant voltage of $.5 \mathrm{~V}$, provided both lowsensitivity dc $(1 \mathrm{~mm}=1 \mu \mathrm{mho})$ and high-sensitivity ac $(1 \mathrm{~mm}=$ $.1 \mu \mathrm{mho}$; time constant $=10 \mathrm{sec}$ ) recordings of skin conductance on adjacent channels of the dynagraph.

Each subject participated in two experimental sessions separated by approximately one week. One session involved the reactiontime task, the other, the vigilance task. Half the subjects performed the reaction-time task in the first session, while the other subjects received the vigilance task first. Both sessions began and ended with 5-min baseline periods during which physiological measures were taken.
In the reaction-time session, subjects received 80 trials of a warned reaction-time task. On each trial a warning tone $(1,000$ or $500 \mathrm{~Hz}, 1-\mathrm{sec}$ duration, 50 -msec rise/fall time, $80 \mathrm{~dB}$ ) was followed after $6 \mathrm{sec}$ by the illumination of a green light. Tones were presented through headphones and a green light was positioned on a panel $3 \mathrm{ft}$ in front of the subject. Intertrial interval varied between 20 and $30 \mathrm{sec}$ (mean $=25 \mathrm{sec}$ ).

The warning stimulus was either a low- $(500-\mathrm{Hz})$ or high$(1,000-\mathrm{Hz})$ frequency tone. For half the subjects, the low tone indicated a go trial and the high tone, a no-go trial. The reverse was the case for the other subjects. The sequence of go and no-go trials was random with the constraint that 40 go and 40 no-go trials occurred during the 80-trial session. The subjects were instructed to respond (by pressing a microswitch held in the dominant hand) as soon as possible after the green light was illuminated if the warning tone had signified a go trial; on no-go trials, they were told to relax. The instructions stressed a quick response, but the subjects were cautioned against responding on no-go trials.

In the vigilance session, the subjects received a series of tone stimuli ( $80 \mathrm{~dB}, 50-\mathrm{msec}$ rise/fall time, 1-msec duration) at regular intervals of $1 \mathrm{sec}$. For most of the session the frequency of the tone was $1,000 \mathrm{~Hz}$, but occasionally it changed to $920 \mathrm{~Hz}$. These "target" tones occurred 12 times during the 36-min session at intervals ranging from 135 to $224 \mathrm{sec}$. The subjects were instructed to indicate detection of the target tones by pressing the microswitch as soon as possible after hearing the stimuli.

\section{Quantification of the Data}

Interbeat intervals (IBI) of heart period activity and respiration amplitude were sampled. Since the cross-spectral analysis assumes that the data are sequences of events equally spaced in time and the responses in the reaction-time task were dependent upon the time of stimulus onset, the beat-to-beat measures were converted into time-based (Porges et al., 1981). Successive 500-msec intervals were established, and an estimate of heart period for each interval was computed as the sum of each heart period that occupied or partially occupied the interval, multiplied by the proportion of the interval that it occupied (see Cheung \& Porges, 1977). Respiration amplitude was sampled at 500 -msec intervals. A measure of spontaneous electrodermal activity was obtained by counting the number of pen deflections reflecting an increase in conductance of at least $.05 \mu \mathrm{mhos}$.

The cross-spectral analysis of heart period activity and respiration amplitude generates a coherence spectrum. The coherence function is analogous to the square of the correlation coefficient and has a range from 0 to 1.0. Coherence reflects the ratio of shared variance between heart period activity and respiration amplitude on each frequency identified on the spectrum. Since the respiratory frequency is not constant, but reflects the dynamic adjustment of the pulmonary system, it is not possible to characterize the coupling between respiration and heart period by calculating the coherence at a specific frequency. A weighted coherence, $C_{W}$, has been calculated to provide an exact measurement of the ratio of heart period variance shared with respiration. The mathematical justification and an elaborate discussion of the method is provided in an earlier publication (see Porges et al., 1980).

In the present experiment, $C_{W}$ was evaluated by the following equation:

$$
\mathrm{C}_{\mathrm{w}}=\frac{\sum_{\theta_{1}}^{\theta_{2}} \mathrm{C}(\theta) \hat{\mathrm{f}}_{\mathrm{H}}(\theta)}{\sum_{\theta_{1}}^{\theta_{2}} \hat{\mathrm{f}}_{\mathrm{H}}(\theta)} .
$$

In the above formula, $\mathrm{C}$, the coherence, reflects the ratio of shared variance between respiration and heart period at each of the fre. 
quencies on the band $\theta_{1}$ to $\theta_{2}$. In the present experiment, $\theta_{1}$ to $\theta_{2}$ includes the frequencies most representative of respiratory rhythm in adults, $\theta_{1}$ is equivalent to 8 cycles $/ \mathrm{min}(.125 \mathrm{~Hz})$, and $\theta_{2}$ is equivalent to 25 cycles/min $(.42 \mathrm{~Hz})$. By multiplying the coherence at each frequency by the spectral density of heart period at each frequency, the amount of heart period variance that is shared with respiration is calculated for each frequency. These estimates of shared variance are summed across the respiratory frequency band, $\theta_{1}$ to $\theta_{2}$, to provide an estimate of the amount of shared variance. To normalize the amount of shared variance into a ratio measure, the quantity is divided by the total variance of the heart rate process across this frequency band (i.e., the sum of the spectral densities). The ratio of shared to total variance across a band of frequencies defines $C_{W}$. In other words, $C_{W}$ is the result of weighting each coherence within the respiratory frequency band by the amount of heart period variance (i.e., spectral density) on the same frequency. Therefore, the coherence occurring on frequencies characterized by large spectral densities for the heart period process will contribute more to $C_{\mathrm{W}}$ than the coherence occurring on frequencies characterized by small spectral densities for the heart period process.

The $C_{W}$ can be calculated with the above arithmetical manipulations on the coherence spectrum derived from most crossspectral analysis packages. In the present study, the cross-spectral analyses were calculated with the BMD package (Dixon, 1970). A discrete Fourier transform was calculated with 25 lags, and a Tukey-Hamming window was used in the frequency domain to smooth the spectrum. To remove linear shifts and other slow complex trends that would violate the assumption of weak stationarity, which is necessary for spectral analysis (Chatfield, 1975), the data set was transformed by calculating successive differences prior to the spectral analysis (see Box \& Jenkins, 1976).

Baselines. For the four 5-min baseline periods, before (pre) and after (post) each of the two tasks, samples of heart period, respiration amplitude, and spontaneous electrodermal activity were obtained. For heart period and respiration, these samples were derived from two 100 -sec periods separated by a 30 -sec interval in the middle of a 5 -min baseline period. The mean heart period and heart period variance were calculated for each 100 -sec epoch. The successive differences for each 100 -sec epoch of heart period and respiration were analyzed by cross-spectral analysis. In each 5 -min rest period, the number of spontaneous electrodermal responses was counted. Estimates of $\mathrm{C}_{\mathrm{W}}$, heart period, and heart period variance were derived for each baseline by taking the average of the two 100 -sec periods.

\section{Reaction Time Task}

On each trial, 10 second-by-second interbeat interval values were derived. The sample began $1 \mathrm{sec}$ before the warning tone and terminated $3 \mathrm{sec}$ after the respond light. For the statistical analysis, the heart period estimate for the prewarning-signal second was subtracted from the succeeding $9 \mathrm{sec}$. Data for go trials and no-go trials were grouped separately and averaged for four trial blocks of 10 trials for each of the two trial types. Reaction time for go trials was obtained to the nearest millisecond.

\section{Vigilance Task}

Twelve estimates of $\mathrm{C}_{\mathrm{W}}$ were calculated from 100 -sec samples taken between each of the 12 target events. The samples began $15 \mathrm{sec}$ after each event.

\section{RESULTS}

\section{Reaction-Time Task}

Subjects were separated at the median into high- $\mathrm{C}_{\mathrm{W}}$ and low- $\mathrm{C}_{\mathrm{W}}$ groups based upon the preexperimental baseline. An analysis of variance of the second-bysecond heart period response pattern exhibited significant seconds $[F(8,144)=7.1, p<.0001], C_{W}$ group

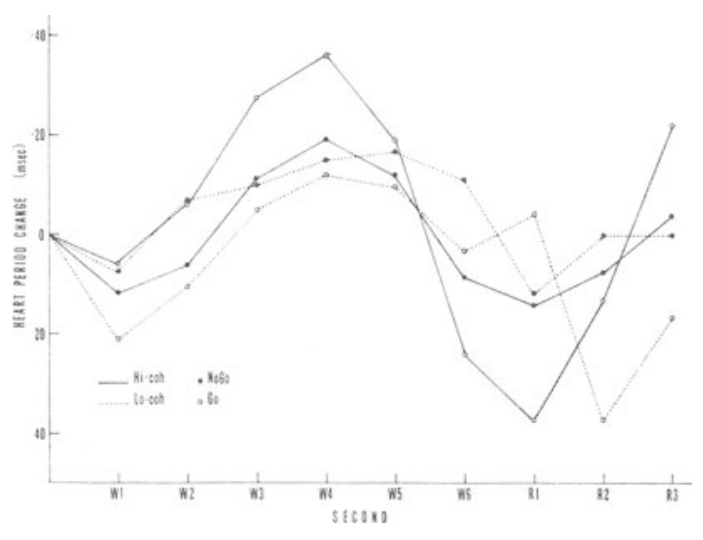

Figure 1. Second-by-second heart period response for the highweighted-coherence (Hi-coh) and the low-weighted-coherence (Lo-coh) groups during the go and no-go trials.

$\times$ seconds $[\mathrm{F}(8,144)=2.0, \mathrm{p}<.05]$, and task $(\mathrm{go} /$ no go) $\times C_{W}$ group $\times$ seconds $[F(8,144)=2.1, p<.05]$ effects and interactions. The task $\times \mathrm{C}_{\mathrm{W}}$ group $\times$ seconds interaction is shown in Figure 1.

As illustrated in Figure 1, there was an obvious difference in the response topography for the two $\mathrm{C}_{\mathrm{W}}$ groups. Simple effects of the interaction identified a significant $C_{W}$ group $\times$ seconds interaction only on the go task and a significant task $\times$ seconds interaction for the high- $\mathrm{C}_{\mathrm{W}}$ group. Further partitioning of the interaction indicated that the only significant response pattern in Figure 1 was exhibited by the high- $\mathrm{C}_{\mathrm{W}}$ group on the go task. The response patterns were not significant on the no-go task for both groups and on the go task for the low- $\mathrm{C}_{\mathrm{W}}$ group. The apparent difference between the two groups in the heart period response in anticipation of the respond signal might be thought to be a function of when the subjects within each group exhibited peak decelera-

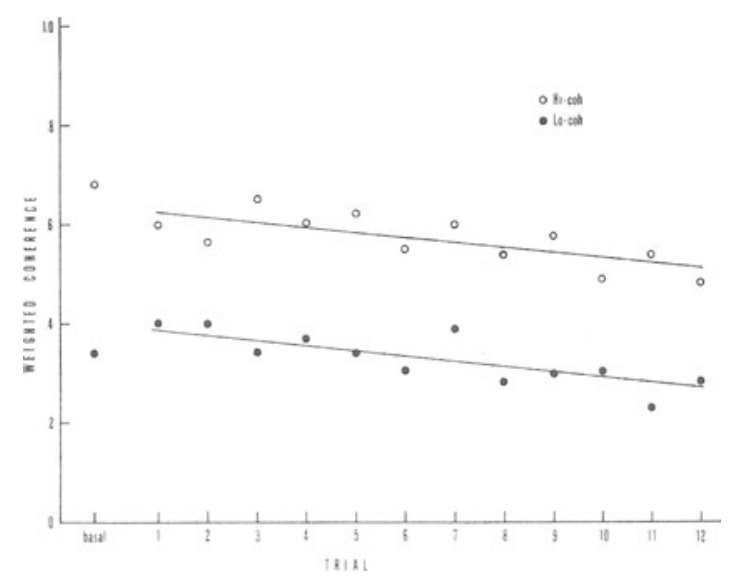

Figure 2. Changes in weighted coherence for the high-weightedcoherence (Hi-coh) and the low-weighted-coherence (Lo-coh) groups during the vigilance task. 
tion, rather than the magnitude of the response. A test was performed selecting the difference between the pretrial heart period and the maximum heart period 5.5 to $9.0 \mathrm{sec}$ after stimulus onset. This analysis, that is, evaluating the magnitude of deceleration independently of "strict" temporal locking to the respond signal, resulted in a nonsignificant interaction, $C_{W}$ group $\times$ task (go/no go) $[F(1,18)=.04]$. There were no differences in the magnitude of deceleration when the deceleration was evaluated across this broader time band. Reaction-time performance was evaluated, but $C_{W}$ group classification was unrelated to performance.

\section{Vigilance Task}

To assess the influence of sustained attention on respiratory-heart rate coupling, $\mathrm{C}_{\mathrm{W}}$ was assessed at 12 time intervals during the vigilance task. The subjects were grouped into high- and low- $C_{W}$ groups based upon the pre baseline period. An analysis of variance indicated only a significant trial effect $[F(11,198)=2.5, p<.005]$. As illustrated in Figure 2 , both groups exhibited a reduction in $\mathrm{C}_{\mathrm{W}}$ as the task progressed. Group classification was not related to performance defined in terms of correct detections. In fact, subjects made few errors during the task.

\section{Stability of Individual Differences Between and Within Experimental Sessions}

To assess the stability of individual differences between and within experimental sessions, correla-

\begin{tabular}{|c|c|c|c|c|c|}
\hline & & \multicolumn{2}{|c|}{ Vigilance } & \multicolumn{2}{|c|}{ Go/No Go } \\
\hline & & Pre & Post & Pre & Post \\
\hline \multirow{3}{*}{ Vigilance } & & \multicolumn{4}{|c|}{ Weighted Coherence } \\
\hline & Pre & 1.00 & & & \\
\hline & Post & $.65 \dagger$ & 1.00 & & \\
\hline Go/No Go & Pre & $.59 \dagger$ & $.58 \dagger$ & 1.00 & \\
\hline Go/1No Go & Post & $.72 \dagger$ & $.53 \dagger$ & $.60 \dagger$ & 1.00 \\
\hline & & \multicolumn{4}{|c|}{ Heart Period } \\
\hline Vigilance & Pre & 1.00 & & & \\
\hline & Post & $.88 *$ & 1.00 & & \\
\hline & Pre & .37 & .40 & 1.00 & \\
\hline Go/No Go & Post & .32 & .37 & $.88 \dagger$ & 1.00 \\
\hline & & \multicolumn{4}{|c|}{ Heart Period Variability } \\
\hline Vigilance & Pre & 1.00 & & & \\
\hline Viglialice & Post & $.47 \dagger$ & 1.00 & & \\
\hline \multirow[t]{3}{*}{ Go/No Go } & Pre & .18 & .37 & 1.00 & \\
\hline & Post & .17 & $.47^{*}$ & $.61 \dagger$ & 1.00 \\
\hline & & \multicolumn{4}{|c|}{$\begin{array}{c}\text { Spontaneous } \\
\text { Skin Conductance Responses }\end{array}$} \\
\hline \multirow{2}{*}{ Vigilance } & Pre & 1.00 & & & \\
\hline & Post & .34 & 1.00 & & \\
\hline \multirow[t]{2}{*}{ Go/No Go } & Pre & $.54 *$ & .26 & 1.00 & \\
\hline & Post & $.70 \dagger$ & .03 & $.58 \dagger$ & 1.00 \\
\hline
\end{tabular}

${ }^{*} p<.05 . \quad t p<.01$.
Table 2

\begin{tabular}{|c|c|c|c|c|}
\hline \multirow[b]{2}{*}{ Variables } & \multicolumn{4}{|c|}{ Correlations } \\
\hline & SCR & HP & HPV & $\mathrm{C}_{\mathrm{w}}$ \\
\hline & \multicolumn{4}{|c|}{ Pre Vigilance } \\
\hline SCR & 1.00 & & & \\
\hline HP & -.23 & 1.00 & & \\
\hline HPV & -.11 & .42 & 1.00 & \\
\hline \multirow[t]{2}{*}{$\mathrm{C}_{\mathrm{w}}$} & .11 & -.15 & $-.55 *$ & 1.00 \\
\hline & \multicolumn{4}{|c|}{ Pre Go/No Go } \\
\hline SCR & 1.00 & & & \\
\hline HP & .21 & 1.00 & & \\
\hline HPV & .10 & .30 & 1.00 & \\
\hline \multirow[t]{2}{*}{$\mathrm{C}_{\mathrm{w}}$} & -.14 & -.15 & $-.63 \dagger$ & 1.00 \\
\hline & \multicolumn{4}{|c|}{ Post Vigilance } \\
\hline SCR & 1.00 & & & \\
\hline HP & -.07 & 1.00 & & \\
\hline HPV & .28 & .36 & 1.00 & \\
\hline \multirow[t]{2}{*}{$\mathrm{C}_{\mathrm{w}}$} & -.09 & .07 & $-.56 \dagger$ & 1.00 \\
\hline & \multicolumn{4}{|c|}{ Post No/Go } \\
\hline SCR & 1.00 & & & \\
\hline $\mathrm{HP}$ & .40 & 1.00 & & \\
\hline HPV & .24 & $.64 \dagger$ & 1.00 & \\
\hline $\mathrm{C}_{\mathbf{w}}$ & -.24 & -.00 & -.40 & 1.00 \\
\hline
\end{tabular}

tions were calculated for skin conductance, heart period, heart period variance (HPV), and $\mathrm{C}_{\mathrm{W}}$. To normalize the HPV measure, the logarithm of heart period variance was employed in the analyses. Correlations were calculated among pre and post baselines for the two experimental sessions. A significant prepost correlation within a task would represent withinsession stability, and a significant pre-pre correlation between tasks would represent between-session stability. Correlation matrices $(n=20, r \geqslant .44, p<.05)$ are presented in Table 1 for heart period (HP), In heart period variance (HPV), frequency of spontaneous skin conductance responses (SCR), and $\mathrm{C}_{\mathrm{W}}$. $\mathrm{C}_{\mathrm{W}}$ exhibited the most consistent correlations, reflecting stability between and within sessions.

The correlations $(n=20, r \geqslant .44, p<.05)$ between physiological variables within each baseline were also assessed and are reported in Table 2 . The only consistent significant correlation was the negative relationship between $\mathrm{C}_{\mathrm{W}}$ and HPV.

\section{DISCUSSION}

The present experiment attempted to relate individual differences in $C_{W}$ to two manifestations of attention: (1) the performance on attention-demanding tasks, and (2) the autonomic response pattern. Both measures have commonly been used as indices of attention (e.g., Coles, 1972; Graham \& Clifton, 1966; Lacey, 1967; Porges, 1972; Walter \& Porges, 1976).

In the reaction-time task, we were interested in behavioral (reaction time) and autonomic (heart rate) aspects of phasic attention. Conversely, in the vig- 
ilance task, the concern was with behavioral (detection) and autonomic $\left(\mathrm{C}_{\mathrm{W}}\right)$ aspects of sustained attention. In neither task was performance related to $C_{W}$ group. In the vigilance task, the failure to find a group-performance relationship may have been due to a ceiling effect (virtually every signal was detected by both groups). In the reaction-time task, the absence of a group difference in performance is less easily explained, because foreperiod cardiac deceleration did discriminate between $\mathrm{C}_{\mathrm{W}}$ groups. Because performance- $\mathrm{C}_{\mathrm{W}}$ relationships were not observed, the mediating role of individual differences in $C_{W}$ on the relationship between autonomic responses and performance (e.g., Duncan-Johnson \& Coles, 1974) was not evident. There are at least three explanations for these findings. First, the $\mathrm{C}_{\mathrm{W}}$ measure may be more sensitive to the individual differences in autonomic responsivity than to performance; second, the relationship between performance and autonomic response patterns may not be reliable; third, performance on the two tasks may not be sensitive to individual differences in attention. Support for the above explanations may be derived from either the literature on heart rate responses and attention or from the data generated in the present experiment. For example, there has been an inconsistency in the literature relating reaction-time performance to the magnitude of heart rate deceleration (Elliott, 1972). Moreover, the construct of attention is complex, and simple reaction-time or vigilance tasks may not be sensitive to the higher order processes that may be related to $\mathrm{C}_{\mathrm{W}}$.

The data from the present study clearly implicate $\mathrm{C}_{\mathrm{W}}$ as an individual difference that is related to the response topography of the anticipatory deceleration, an autonomic response associated with attention and orienting (e.g., Graham \& Clifton, 1966). The relationship between autonomic responsivity and performance may change as task complexity is manipulated (e.g., Walter \& Porges, 1976). Thus, one may speculate that the current finding of dissociation between performance levels on both of the attentiondemanding tasks and individual differences in spontaneous autonomic activity (i.e., $\mathrm{C}_{\mathrm{W}}$ ) or autonomic reactivity (i.e., anticipatory deceleration) may be directly related to the constricted range of performance levels during both tasks.

This paper reports the first demonstration that the degree of coupling between cardiac and respiratory activity measured with the weighted coherence procedure is a relatively stable individual difference characteristic. $\mathrm{C}_{\mathrm{W}}$ exhibited the largest within- and betweensession correlations, of all physiological measures obtained during the baseline periods. The betweensession correlations reflected stability over approximately a 1 -week interval.

The reaction-time data reveal that the $C_{W}$ groups differed in heart period response pattern on trials on which the warning stimulus indicated that a response to the imperative stimulus would be required. In fact, only the high- $\mathrm{C}_{\mathrm{W}}$ group exhibited a significant response on these trials. When the groups were compared with respect to magnitude of deceleration defined in terms of a loose temporal criterion, no differences were found. This suggests that the difference between $\mathrm{C}_{\mathrm{W}}$ groups lies in the timing, rather than the magnitude, of the decelerative response. If the anticipatory deceleration is interpreted as a temporally conditioned heart rate response (Porges, 1972), then $C_{W}$ may reflect, as suggested earlier in the paper, individual differences in conditionability. Temporal contiguity is generally accepted as an underlying requisite condition for conditioning. In the classical conditioning paradigm, contiguity implies that the time interval between the conditioned stimulus and the unconditioned stimulus is constant. In the fixed-foreperiod reaction-time task, anticipatory deceleration may be viewed as a conditioned response (see Porges, 1972). $C_{W}$ may be a physiological manifestation of nervous system "contiguity," since $C_{W}$ reflects the contiguity between the respiratory input and heart period modulation across the band of respiratory frequencies. Thus, the demonstration that only the high- $\mathrm{C}_{\mathrm{W}}$ subjects responded with an anticipatory deceleration in the fixed-foreperiod reactiontime implies that $C_{W}$ may reflect an important organismic determinant of heart rate conditioning.

\section{REFERENCES}

Box, G. E. P., \& Jenkins, G. M. Time series analysis, forecasting and control. San Francisco: Holden Day, 1976.

Brillinger, D. R. Time series data analysis and theory. Chicago: Holt, Rinehart \& Winston, 1975.

Chatfield, C. The analysis of time series: Theory and practice. London: Chapman \& Hall, 1975.

Cheung, M. N., \& Porges, S. W. Respiratory influences on cardiac responses during attention. Physiological Psychology, 1977, 5, 53-57.

Coles, M. G. H. Cardiac and respiratory activity during visual search. Journal of Experimental Psychology, 1972, 96, 371-379.

Dixon, W. T. BMD-biomedical computer programs. Los Angeles: University of California Press, 1970.

Duncan-Johnson, C. C., \& Coles, M. G. H. Heart rate and disjunctive reaction time: The effects of discrimination requirements. Journal of Experimental Psychology, 1974, 103, 1160-1168.

Elliot, R. The significance of heart rate for behavior: A critique of Lacy's hypothesis. Journal of Personality and Social Psychology, 1972, 22, 398-409.

Graham, F. K., \& Clifton, R. K. Heart rate changes as a component of the orienting response. Psychological Bulletin, 1966, 65, 305-320.

LACEY, J. I. Somatic response patterning and stress: Some revisions of activation theory. In M. H. Appley \& R. Trumbell (Eds.), Psychological stress: Issues in research. New York: Appleton-Century-Crofts, 1967.

Lopes, O. U., \& Palmer, J. F. Proposed respiratory 'gating' mechanism for cardiac slowing. Nature, 1976, 264, 454-456.

Porges, S. W. Heart rate variability and deceleration as indexes 
of reaction time. Journal of Experimental Psychology, 1972, 92, 103-110.

Porges, S. W. Individual differences in attention: A possible physiological substrate. In B. K. Keogh (Ed.), Advances in special education. Greenwich, Conn: JAI Press, 1980, 111-134.

Porges, S. W., Bohrer, R. E., Cheung, M. N., Drasgow, F., McCabe, P. M., \& Keren, G. New time-series statistic for detecting rhythmic co-occurrence in the frequency domain: The weighted coherence and its application to psychophysiological research. Psychological Bulletin, 1980, 88, 580-587.

Porges, S. W., Bohrer, R. E., Keren, G., Cheung, M. N., Franks, G. J., \& Drasgow, F. The influence of methylphenidate on spontaneous autonomic activity and behavior in children diagnosed as hyperactive. Psychophysiology, 1981, 18, 42-48.
Porges, S. W., McCabe, P. M., \& Yongue, B. G. Respiratoryheart rate interactions: Psychophysiological implications for pathophysiology and behavior. In J. Cacioppo \& R. Petty (Eds.), Perspectives in cardiovascular psychophysiology. New York: Guilford Press, 1982.

Walter, G. F., \& Porges, S. W. Heart rate and respiratory responses as a function of task difficulty: The use of discriminant analysis in the selection of psychologically sensitive physiological responses. Psychophysiology, 1976, 13, 563-571.

(Manuscript received August 25, 1981; revision accepted for publication February 10, 1982.) 